\title{
The Effect of Blood Pressure Pulsatility on the Baroreflex Gain
}

\author{
Méabh Loughman ${ }^{1,2,3}$, Annraoi de Paor $^{4}$, John V. Ringwood ${ }^{1}$ \\ Department of Electronic Engineering, Maynooth University, Co. Kildare ${ }^{1}$, \\ The Royal College of Surgeons in Ireland, Dublin ${ }^{2}$, \\ Dublin City University, Dublin ${ }^{3}$,University College Dublin, Dublin ${ }^{4}$
}

\begin{abstract}
This paper makes a connection between blood pressure pulsatility and baroreflex gain in human subjects. While baroreflex gain may be difficult to examine, low frequency oscillations found in human blood pressure can be used as a surrogate coarse measurement of the baroreflex gain. Analysis is performed via a mathematical model that examines the presence or absence of a low frequency oscillation in human blood pressure signal. Mayer waves are well-known low frequency oscillations that occur in human blood pressure signals at approximately $0.1 \mathrm{~Hz}$. This paper proposes that an examination of Mayer waves can be used to examine variation in baroreflex gain. This examination could provide more information on the effect of an absence of pulsatility in left ventricular assist device (LVAD) patients.
\end{abstract}

\section{INTRODUCTION}

Mean Arterial Pressure (MAP) is the mean value of blood pressure (BP). BP is regulated by short and long term physiological feedback loops. Neural control is prominent throughout this homeostatic process. Baroreceptors are stretch receptors found in the carotid sinus and the aortic arch. In the most simplified sense the baroreflex can be thought of as a feedback model with four components. A detector (Bareceptors), an Integration Centre (the Medulla Oblangata), the controller (the autonomic nervous centre) and the effectors (the vasculature and blood vessels). The input is an impulse of information traveling from the baroreceptor, which is triggered by the MAP. The output from the controller effects both the sympathetic nervous system and the parasympathetic nervous system. The parasympathetic nervous system causes affects in the sinoatrial node, leading to a reduction in heart rate (HR). The sympathetic nervous system causes an opposed effect on HR. The sympathetic nervous system also

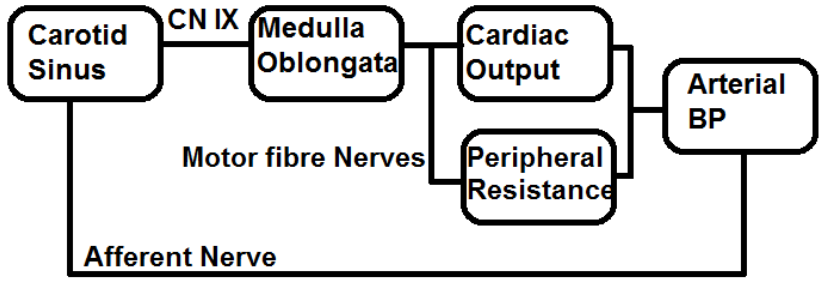

Figure 1. Components of the sympathetic baroreflex effected by the baroreceptors located in the carotid sinus

has an effect on the ventricular myocardium, increasing contractility, and the arterioles, increasing resistances and the veins increasing vasomotor tone [1].

In anatomical terms this papers primary focus is on the sympathetic baroreflex altered by the baroreceptors located in the carotid sinus. Figure 1 references the pathway in which the impulse travels by means of electromechanical transduction. Arterial pressure is converted into nerve activity by the baroreceptors located on either side of the carotid sinus [2]. Sensory nerves conduct impulses along the glossopharyngeal nerve (CN IX) to the Medulla Oblongata, where the Nucleas Tractus Solartis converts the impulse into a motor nerve activity impulse that is then conducted along sympathetic nerve fibres. The sympathetic nerves alters peripheral resistance and HR. Altering heart rate and contractility of organs alters the cardiac output $(\mathrm{CO})$. The product of $\mathrm{CO}$ and peripheral resistance is aterial BP. Figure 1 represents the baroreflex examined by this paper.

This paper contends that, through simulation and analysis of the Mayer wave conduction times of 


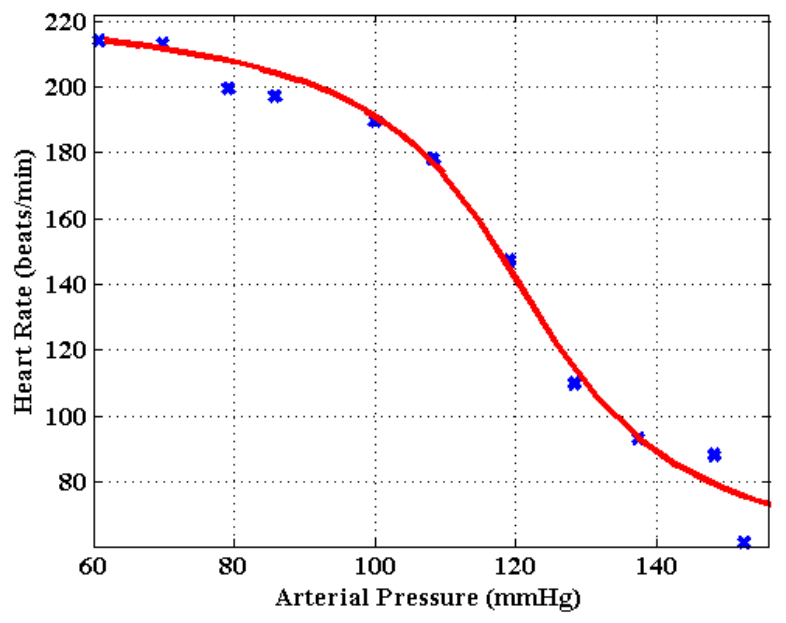

Figure 2. Heart rate as a function of mean arterial pressure

efferent and afferent delays, BP pulsatility is responsible for the low frequency oscillation (LFO) known as the Mayer wave.

\section{The BARoreflex Curve}

A complex relationship between components of the baroreflex can be described by a sigmoidial relationship known as the baroreflex curve. The baroreflex curve is a phenomena that relates $\mathrm{BP}$ and other physiological quantities such as HR and sympathetic nerve activity. Previous works [3][4] utiltise the baroreflex curve phenomena in order to model the baroreflex. In [4] the authors conclude that Mayer waves are sometimes present in the BP of rabbits utilising the baroreflex curve component to model the baroreflex. The LFO has different frequency values across species sizes. This is proposedly due to the diverse lengths of nerves the nerve impulse must travel along [4] which depend on the size of the species. Human Mayer waves are known to occur at approximately $0.1 \mathrm{~Hz}$.

The baroreflex curve can be estimated utilising typical values from [5]. It is possible to use HR as a coarse measurement of the baroreflex curve due to the aforementioned sigmoidial relationship. The sigmoidial curve can be described mathematically by equation 1, given by [6], and can be seen in Figure 2.

$$
\left.y=h \tan ^{-1}\left(\beta\left(x-x^{*}\right)\right)+y^{*}\right)
$$

The parameters $\beta, h, x^{*}$ and $y^{*}$ specify the shape and position of the characteristics of the baroreflex curve. $\beta$ denotes curvature of the sigmoid, $x^{*}$ and $y^{*}$ are the horizontal and vertical centre points of the sigmoid. $x^{*}$ and $y^{*}$ can be seen to be approximately -119 and 142 respectively in Figure 2 . $h$ can also be seen to be half of the vertical range of heart rate.

\begin{tabular}{|c|c|c|c|}
\hline$h$ & $x^{*}$ & $y^{*}$ & $\beta$ \\
\hline 57.89 & -121 & 139 & 0.06 \\
\hline
\end{tabular}

III. A NON-LINEAR MODEL FOR THE BAROREFLEX

In [4] a non-linear model was hypothesized to simulate the baroreflex, which includes the effects of vasculature and the central nervous system (CNS). The model provided by [7] represents the effect of the CNS as a gain $k_{p}$, and the non-linear baroreflex curve function $N(a)$. The non-linear model can be seen in Figure 3.

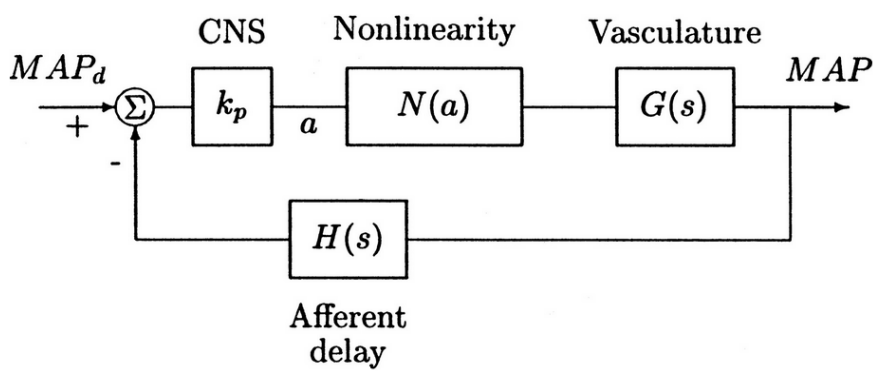

Figure 3. Model of the non-linear baroreflex feedback system

$$
G(s)=\frac{e^{-s \tau_{e}}}{1+s \tau_{v}} \quad, \quad H(s)=e^{-s \tau_{a}}
$$

The pure time delays, $\tau_{a}$ and $\tau_{e}$, are due to conduction time along the efferent and afferent nerves and neurotransmission. $\tau_{v}$ is the vasculature lag. [7] hypothesizes that the conduction time will alter due to the size of species which allows for the hypothesis that human subjects of different heights may have slightly altered Mayer wave frequency values. This will be discussed further in Section VI.

According to [1] there is a 260-ms average time lag between a sudden increase in carotid sinus pressure 
and inhibition of renal nerve activity. Stimulation of the carotid sinus nerve may not respond for up to 20 s. The response may be greater when a stimulus is applied during low HR, i.e a baroreceptor stimulus does not always produce the same reflex vagal activation [2]. It is notable that an unexplained latency discrepancy is mentioned in [2].

Examining the model in Figure 3, it is evident that pulsatility is not present. To account for a pulsatile component the model must be altered as in [7], which can be seen in Figure 4.

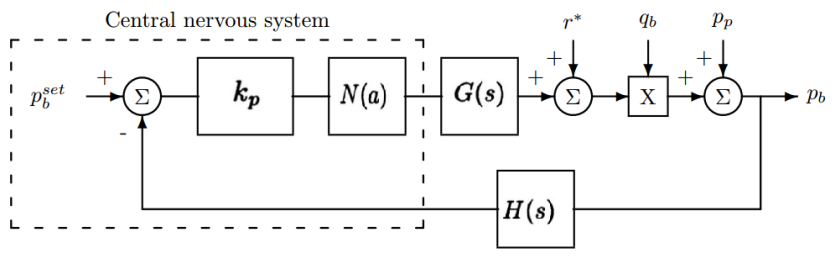

Figure 4. Pulsatile model of baroreflex

In Figure $4, r^{*}$ is the baseline peripheral resistance. The range of peripheral resistance of a human is given by [8] as $700 \rightarrow 1600$ dynes $/ \mathrm{sec} / \mathrm{cm}^{5}$, which may be converted to approximately $8 \rightarrow 20 \mathrm{~mm} \mathrm{Hg}$. $q_{b}$ represents $\mathrm{CO}$, noted to fluctuate between $3.5 \rightarrow$ $5.5 \mathrm{l} / \mathrm{min}$ [8]. $p_{p}$ represents the pulsatile component of the blood pressure signal.

\section{MAYER WAVES}

Previous works [7] have examined pulsatile BP signals in rabbits. Utilizing the MIT-BIH Polysomnographic Database [9], a collection of recordings of multiple physiological signals during sleep, a portion of a human BP signal can be seen in Figure 6. This patient was a 33 year old male with sleep apnea syndrome, it can be seen that the patient is pre-hypertensive due to the abnormal diastolic pressure (approx 70mmHg).

As can be seen in Figure 5, there is activity at approximately $0.13 \mathrm{~Hz}$. According to [7], the Mayer wave may be affected by the length of the conducting afferent and efferent nerve pathways of an individual. A frequency of $0.13 \mathrm{~Hz}$ would indicate shorter nerve lengths.

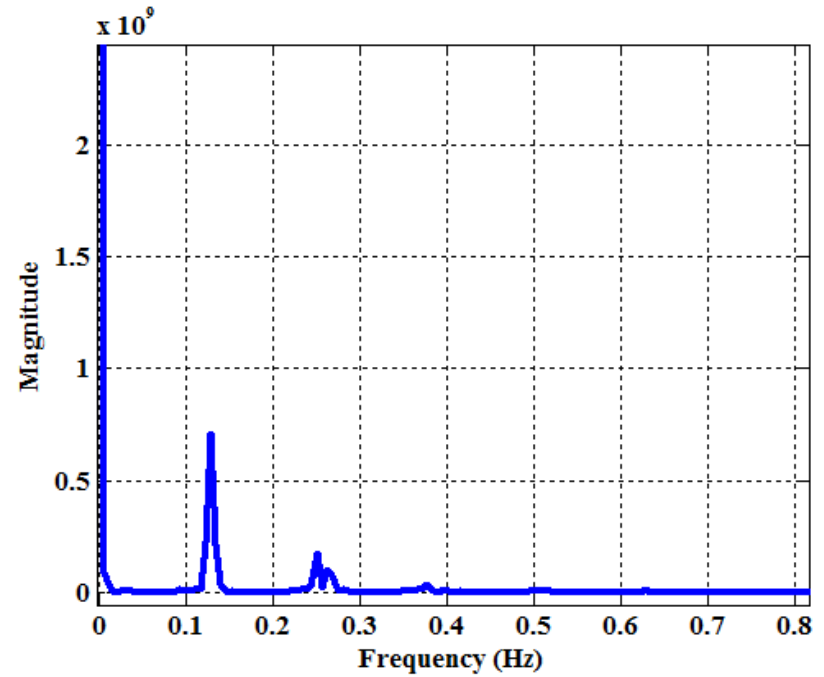

Figure 5. Blood pressure power spectral density

The Mayer wave is considered to be a limit cycle as it is hypothesized to be a self-sustaining oscillation produced by the non-linear model of the baroreflex. The presence, or absence, of the Mayer wave will be discussed further in Section VI.

\section{Puls atility FROM BP Signals}

As in previous work [7] the pulsatile BP signal is broken into five distinct component parts $t_{1}, t_{2}, t_{3}, t_{4}, t_{5}$, see Figure 6 . The method of approximation used in [7] has been utilized for this study in order to model pulsatility of a human $\mathrm{BP}$ signal. The $\mathrm{BP}$ signal is considered to be a dither signal (i.e. an injection of a high frequency signal superimposed on an otherwise low frequency signal). The application of a dither signal allows us to represent the human BP signal by a slowly varying value $r$ and a high frequency dither signal $d(t)$.

[7] utilises the concept of equivalent non-linearity to determine the effect of a combination of a high frequency dither signal (i.e BP) and a non-linearity (i.e baroreflex respsonse). The concept can be seen in Figure 7.

The equivalent non-linearity of the baroreflex can be calculated as the sum of the distinct component parts seen in Figure 6. 


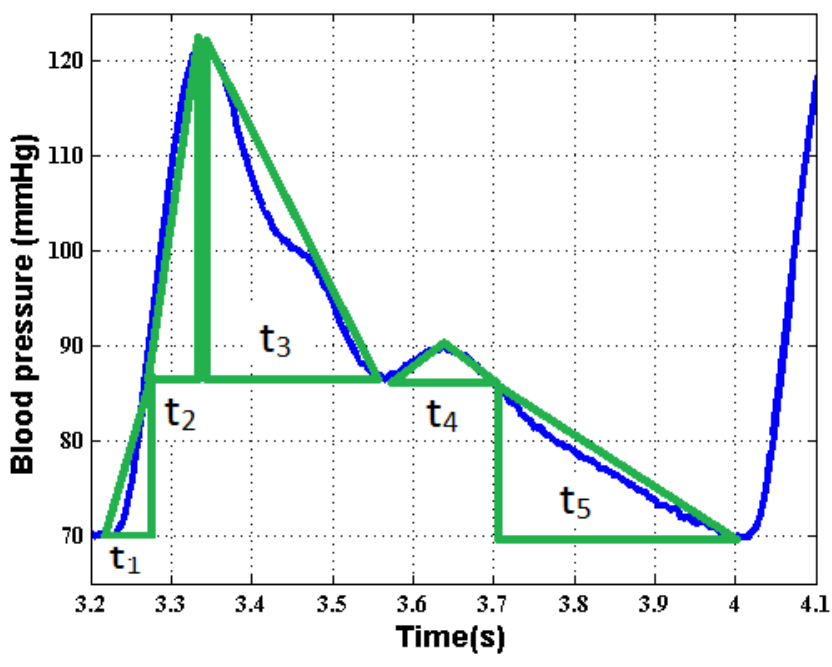

Figure 6. Human BP signal accessed at [9]

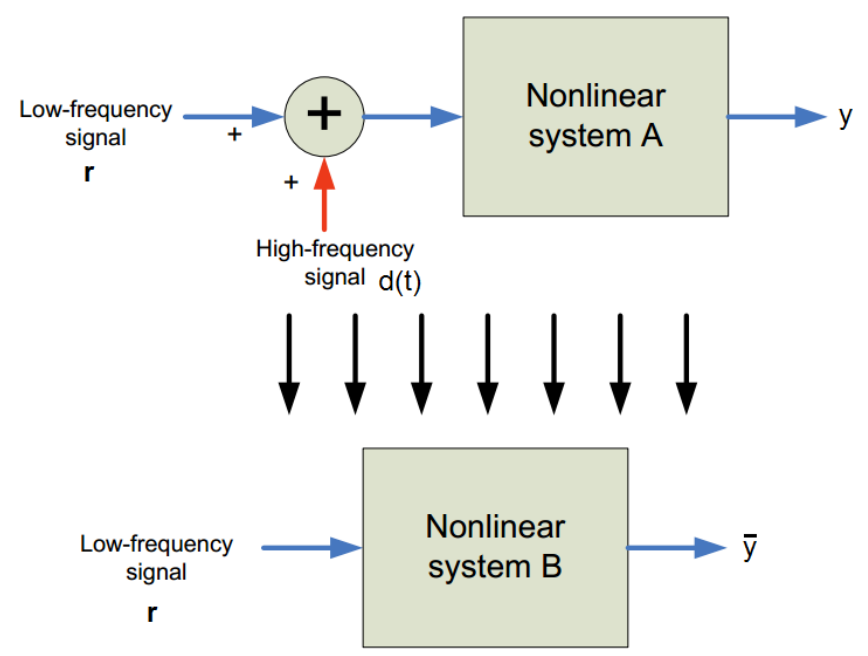

Figure 7. Equivalent non-linearity concept

The baroreflex curve given by Equation 1 can be seen plotted against the equivalent non-linearity of the pulsatile baroreflex system, as in Figure 8. This was perforemd substituting human parameters into the equivalent non-linearity, originally parametrized for rabbits by [7]. It can be seen in Figure 8 that there is a large increase in baroreflex gain in the absence of pulsatile BP signal, approximately $33 \%$.

\begin{tabular}{|c|c|c|}
\hline$\tau_{e}(s)$ & $\tau_{a}(s)$ & $\tau_{v}(s)$ \\
\hline 3 & 0.01 & 5 \\
\hline
\end{tabular}

The values in Table $\mathrm{V}$ are the combinations of $\tau_{v}, \tau_{a}$ and $\tau_{e}$ used for the analysis that most closely match human physiology.

The equivalent non-linearity of the baroreflex was determined for a single-valued non-linearity, $f()$, with input $u(t)=r+d(t), d(t)$ being the dither signal [7].

$$
y=f(r+d(t))
$$

Letting $p(q) d q$ be the probability that, for any time $t, d(t)$ lies in the range $q$ to $q+d q$, with $p(q)$ being the probability density function for the dither. When $d(t)$ has the value $q$, the expected value for $\mathrm{y}$ is [7]:

$$
\bar{y}=\int_{-\infty}^{\infty} f(r+q) p(q) d q
$$

To calculate $p(q)$, let $F(q)$ be the probability that $d(t)$ lies above $q$, giving

$$
\frac{d F(q)}{d q}=-p(q)
$$

As in [7], all of the probability density functions of the equivalent non-linearity can be expressed as

$$
p_{q}=\frac{d F(q)}{d q}=\frac{1}{2 A_{n}}
$$

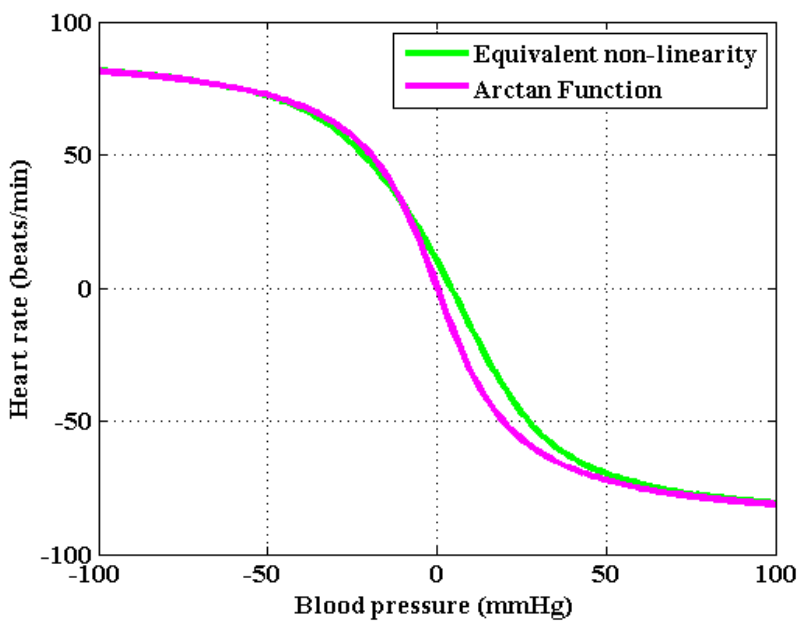

Figure 8. Equivalent non-linearity 
where $A_{n}$ is the maximum amplitude of the dither signal. The equivalent non-linearity of component 1 is:

$$
\left.\overline{y_{n}}=G_{n}(r)=\int_{-\infty}^{\infty} \frac{1}{2 A_{n}}\left[h \tan ^{-1}(\beta(r+q))+y^{*}\right)\right]
$$

Finally the overall equivalent non-linearity of each dither signal can be described as:

$\bar{y}=G(r)=\alpha_{1} G_{1}+\alpha_{2} G_{2}+\alpha_{3} G_{3}+\alpha_{4} G_{4}+\alpha_{5} G_{5}$

The weighting factors $\alpha_{i}$, are the portions of the total period occupied by the $i^{t h}$ pulsatile component.

\section{LFO CASE ANALYSIS}

A number of physiological subsystems exhibit sustained oscillations which are thought to be limit cycles [6]. Limit cycles are consequences of a feedback system itself. In the case of the blood pressure, Mayer waves are thought to be the limit cycle. Mayer waves have been noted to occur in rabbits [7] at a frequency of approximately $0.3 \mathrm{~Hz}$, notably due to the latencies associated with shorter nerve lengths. Mayer waves occur at approximately $0.1 H z$, in human subjects.

In order for Mayer waves to be present the afferent, efferent time delays and vasculature lags must satisfy the equation given by [4]:

$$
p\left(\omega_{o}\right)=-\pi+\omega_{o}\left(\tau_{e}+\tau_{a}\right)+\tan ^{-1}\left(\omega_{o} \tau_{v}\right)
$$

The oscillation frequency of the Mayer wave is determined solely by $\tau_{e}, \tau_{a}$ and $\tau_{v}$ at point, $p$. The location at which $p$ crosses the horizontal axis determines the frequency of the LFO. Simplifying the equation 5 to equation 6 ensures this requirement is achieved.

$$
-\pi=\omega_{o}\left(\tau_{e}+\tau_{a}\right)+\tan ^{-1}\left(\omega_{o} \tau_{v}\right)
$$

Solving for Equation 6 for possible combinations of $\tau_{e}, \tau_{a}$ and $\tau_{v}$, corresponding to the net lag around the

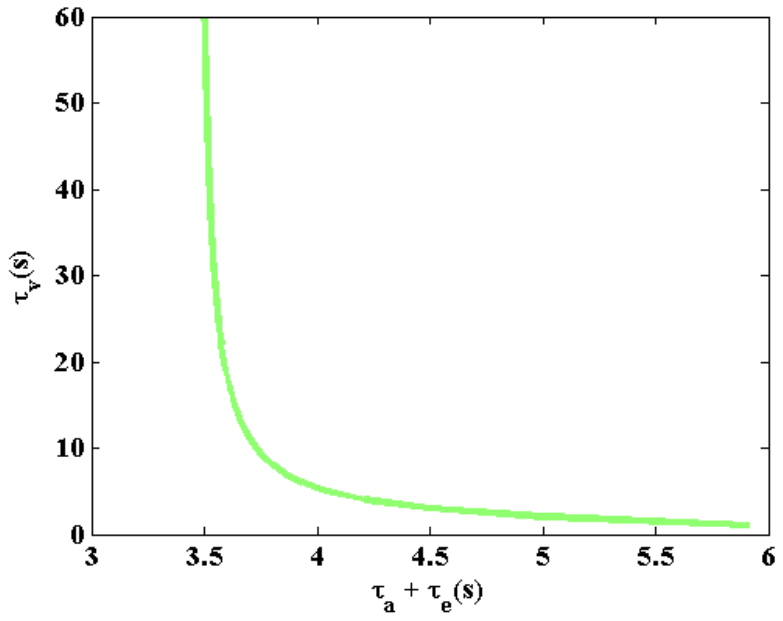

Figure 9. Combinations of neurotransmission time delays and vasculature lags to produce Mayer wave of $0.1 \mathrm{~Hz}$

system in Figure 9, must be $0.1 \mathrm{~Hz}$ and sustained oscillations.

The use of the describing functions (DFs) allow for subsequent frequency domain analysis. The asymptotic value for each of the DFs given by [6] is paramount. DFs are used to express non-linear elements as non-linear gains, by estimating the output fundamental sinusoid amplitudes and phase versus the input sinusoids amplitude and phase, omitting the harmonics of such frequencies. In this paper, DFs are utilised as tools for predicting nonlinear system oscillations. The values of $\beta$ and $h$ are expected to vary for cases of different individuals.

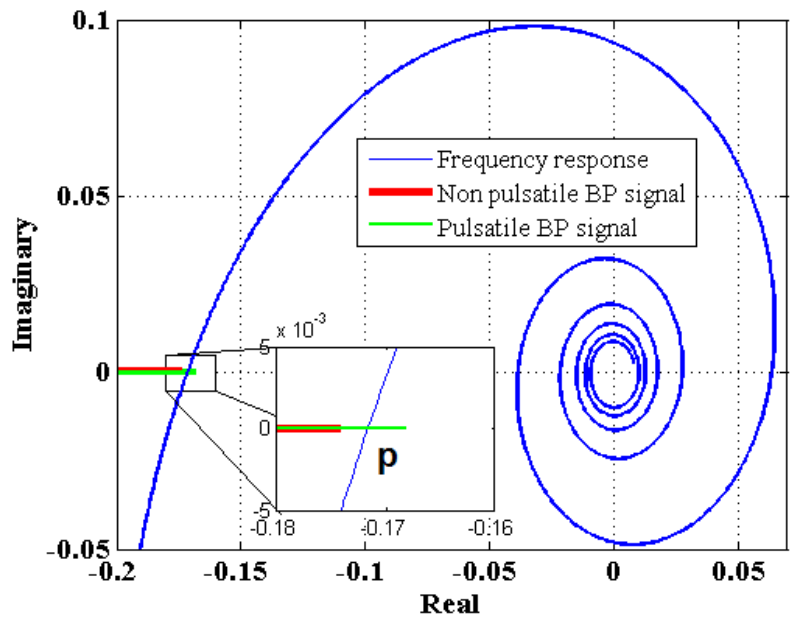

Figure 10. Frequency response using human parameters 


\section{Model AnAlysis}

Using the model seen in Figure 4 to include and omit pulsatility it can be seen that sustained oscillations are preesent in the non-pulsatile case.

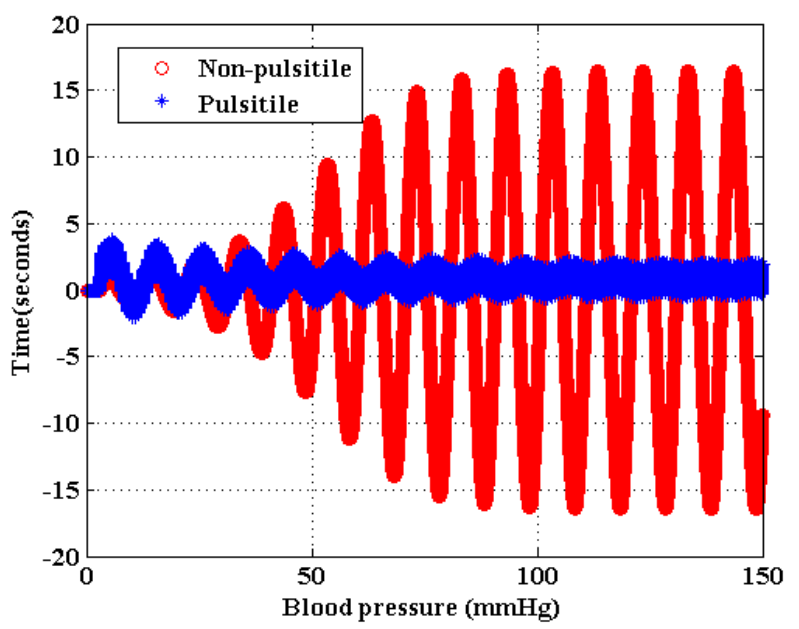

Figure 11. Model of system including and ommiting pulsatility

The pulsatile model has a decaying output without oscillations. The non-pulsatile model has sustained frequency presence at $0.1 \mathrm{~Hz}$ which can be seen in Figure 11. Both the pulsatile model and nonpulsatile model used a gain of 20 .

\section{CONCLUSION}

By comparing the equivalent non-linearity of a pulsatile blood pressure signal and the orignal $\tan ^{-1}$ function given by 1 , it can be seen that there is a gain increase (33\%) in the equivalent nonlinearity calculation of the system when pulsatility is neglected. This can be seen in the area between the saturation limits in Figure 10 and in the limits of the DF functions in Figure 10. This paper contends that a lack of pulsatility results in conditions for a limit cycle to be met which, in turn, provides evidence of an increased baroreflex gain.

As a result, we should expect an increased incidence of low-frequency blood pressure oscillations in patients with LVADs. To this end, en experimental study is currently being carried out at the Mater Misericordiae University Hospital,Dublin to measure blood pressure time series in LVAD patients, both pre- and post-operation, to examine for increases in Mayer wave activity following LVAD insertion.

This paper considers only the neural control of BP using modulation of the peripheral resistance. Neural control of BP is also effected using modulation of heart rate and further studies will examine the effect of pulsitility on both cardiac and peripheral resistance branches operating in tandem.

\section{REFERENCES}

[1] D. L. Eckberg and P. Slight, Human Baroreflexes in Health and Disease. Oxford: Clarendon Presss, 1992.

[2] P. B. Persson and H. R. Kirchheim., Baroreceptor Reflexes. Springer, 1991.

[3] D. E. Burgess, J. C. Hundley, S. G. Li, D. C. Randall, and D. R. Brown, American Journal of Physiology, no. 6 Pt 2.

[4] J. V. Ringwood and S. C. Malpas, "Slow oscillations in blood pressure via a nonlinear feedback model." American Journal of Physiology. Regulatory, integrative and comparative physiology, vol. 280, no. 4, pp. R1105-R1115, 2001.

[5] B. M. K. Berne, Robert M and B. A. Stanton, Berne and Levy Physiology, Sixth Edition. Mosby/Elsevier, 2010.

[6] A. de Paor and J. Ringwood, "A simple soft limiter describing function for biomedical applications." IEEE transactions on biomedical engineering, vol. 53, no. 7, pp. 1233-40, 2006.

[7] J. V. Ringwood, F. Taussi, and A. M. de Paor, "The effect of pulsatile blood flow on blood pressure regulatory mechanisms," 2012 IEEE International Conference on Control Applications, pp. 609-614, 2012.

[8] K. D. Vick, The Washington Manual of Surgery, 5th Ed. Wolters Kluwer/Lippincott Williams Wilkins Health, 2008, vol. 248, no. 5 .

[9] A. L. Goldberger, L. A. N. Amaral, L. Glass, J. M. Hausdorff, P. Ch, R. G. Mark, J. E. Mietus, G. B. Moody, C.-k. K. Peng, H. E. Stanley, and P. C. Ivanov, "PhysioBank, PhysioToolkit, and PhysioNet," Circulation, vol. 101, no. 23, pp. E215-20, 2000. 